\title{
Computer-Aided Assessment of Anomalies in the Scoliotic Spine in 3-D MRI Images
}

\author{
Florian Jäger ${ }^{1}$, Joachim Hornegger ${ }^{1}$, Siegfried Schwab ${ }^{2, \star}$, and Rolf Janka ${ }^{2, *}$ \\ 1 Department of Computer Science, Chair of Pattern Recognition and Erlangen \\ Graduate School in Advanced Optical Technologies (SAOT), \\ University of Erlangen, Germany \\ jaeger@informatik.uni-erlangen.de \\ 2 Radiologic Institute, University of Erlangen, Germany
}

\begin{abstract}
The assessment of anomalies in the scoliotic spine using Magnetic Resonance Imaging (MRI) is an essential task during the planning phase of a patient's treatment and operations. Due to the pathologic bending of the spine, this is an extremely time consuming process as an orthogonal view onto every vertebra is required. In this article we present a system for computer-aided assessment (CAA) of anomalies in 3-D MRI images of the spine relying on curved planar reformations (CPR). We introduce all necessary steps, from the pre-processing of the data to the visualization component. As the core part of the framework is based on a segmentation of the spinal cord we focus on this. The proposed segmentation method is an iterative process. In every iteration the segmentation is updated by an energy based scheme derived from Markov random field (MRF) theory. We evaluate the segmentation results on public available clinical relevant 3-D MRI data sets of scoliosis patients. In order to assess the quality of the segmentation we use the angle between automatically computed planes through the vertebra and planes estimated by medical experts. This results in a mean angle difference of less than six degrees.
\end{abstract}

\section{Introduction}

MRI is being used increasingly to investigate children with scoliosis. Although there may be a hereditary component to true idiopathic scoliosis, the condition has no known cause and is not associated with dysraphism. However, in the infantile and juvenile age group the incidence of spinal cord anomalies like tethered cord, syringomyelia, Chiari malformations, diastematomyelia and meningocele / myelomeningocele ranges from 17.6 to $26 \%$ [12]. Furthermore there can be structural changes of the vertebral bodies like wedge vertebra or hemivertebra. As MRI can visualize all these abnomalities it can be extremely important in the pre-operative planning of scoliosis. Failure to detect abnormalities of the

\footnotetext{
* The authors gratefully acknowledge funding of the Erlangen Graduate School in Advanced Optical Technologies (SAOT) by the German National Science Foundation (DFG) in the framework of the excellence initiative.
} 
neuraxis prior to treatment of scoliosis, particularly with instrumentation that lengthens the spine, can have serious neurological consequences.

With the introduction of 3-D spin echo sequences (SPACE, Siemens, Erlangen, Germany) MRI of the scoliotic spine can be acquired with only two sequences (upper spine and lower spine). However, due to the extreme bending of the vertebral column in all three axes the manual assessment of the spine is a very time consuming process. In some cases it is even impossible for the radiologist to analyze pathological changes within the spine manually. Furthermore, it can be very difficult to specify the anatomic localization of the viewed vertebra.

In this article we introduce a framework for CAA of the spine. We show that it is possible to statistically model the spinal channel and cord. Using this model we perform a segmentation of the spinal channel and cord. Upon this we build an application that enables the physician to assess the scoliotic spine nearly as fast and precise than a non-scoliotic spine which should improve the pre-operative work-up of this young patient group.

Most state-of-the-art methods for the localization of the spine in tomographic images do a segmentation of the vertebra (e.g. 3]). In general, these approaches use assumptions about the spinal appearance that are not fulfilled in data sets of scoliosis patients. Particulary the shape of the scoliotic spine is altered considerably. Thus, all assumptions concerning the typical "s" shape and with that the orientation of the vertebra are no longer valid. Additionally, the shape of the vertebra can vary in a wider range than in the non-scoliotic case. Two typical examples of spinal images of scoliosis patients are shown in Figure 1, Additionally, there are a few methods for segmentation of the spinal cord [45]. The computation of the centerline presented in this article does not depend on any prior information about the shape of the spine nor on the shape of the vertebra. Further on, it is not restricted to the used MRI protocol but can be adapted to other modalities in a straight forward manner. The only requirement is that the spinal channel or the spinal cord are visible within the images.

\section{Method}

The system for the CAA of spine anomalies can be separated into four parts pre-processing of the data sets, the segmentation of the spinal channel/cord, the labeling of the vertebra and finally the visualization of the data.

\subsection{Pre-processing}

Our experiments show that it is enough to use the following standard stateof-the-art pre-processing methods. In order to be able to directly use signal intensities additional to structural components within the images, we apply Homomorphic Unsharp Masking (HUM). This compensates the influence of coil inhomogeneities during the acquisition of the 3-D MRI images. The kernel size used was about $30 \mathrm{~mm}$ [6. Sequentially, we use a signal intensity standardization approach to correct inter-scan intensity variations within the data sets. The 

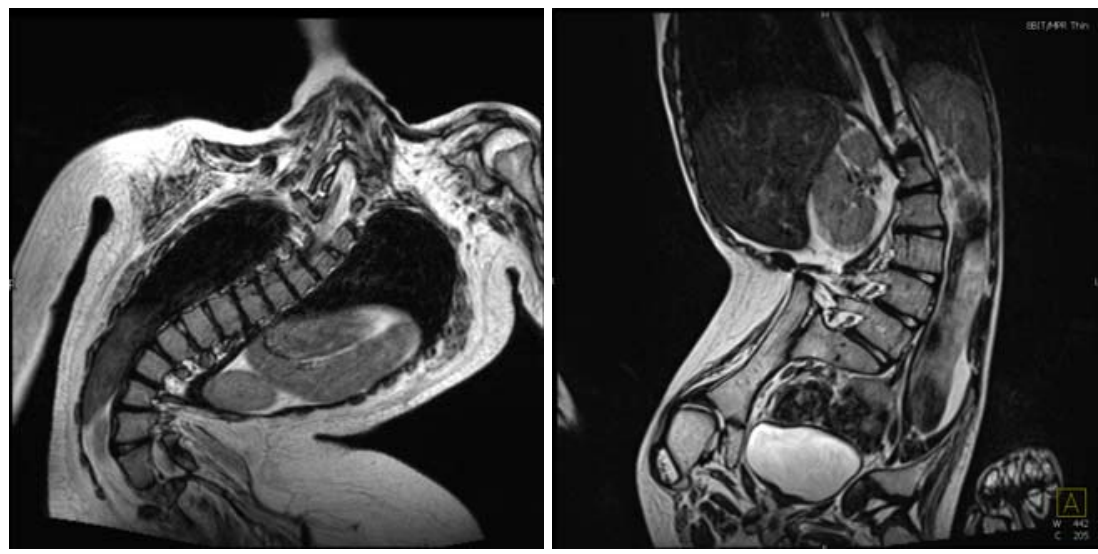

Fig. 1. Coronar slices from two 3-D MRI spine images showing typical scoliotic spines

method used is based on the non-rigid alignment of image histograms [7. Finally, a median filter is applied to the data sets to reduce noise present in the images. All these methods can be applied during the segmentation step on a per voxel basis. Thus, only voxels that are required for the segmentation of the spinal channel/cord are processed. This yields a reduced computational cost.

\subsection{Segmentation Method}

The proposed method for the segmentation of the spinal channel and cord is an iterative process. The basic idea is that in each iteration step the segmentation is propagated with respect to the minimization of an energy function. This function is based on local signal intensities as well as local structural information like image gradients and the distance to the current approximation of the spinal centerline. The spatial coherence is statistically modeled by posteriori probabilities that can easily be formulated as Gibbs distributions.

Initialization Step. For the initialization of the presented method the radiologist has to set a seed point within the spinal channel. From this seed point an adaptive region growing is started. The upper and lower intensity boundary of the segmentation is increased/decreased by one in each growing step until a defined number of voxels $N_{0}$ is contained within the segmented region. Afterwards we apply morphologic closing to the initial segmentation $S_{0}$ to fill potential holes due to signal intensity variations within the images. Heuristics show that $N_{0}=300$ is enough. All segmentation $S_{i}$ are binary images with a value of one for voxels within the segmented region and zero as background value.

Iteration Step. First, in every iteration $i$ the centerline $c_{i}$ is approximated using the segmented region $S_{i-1}$ from the previous iteration. The estimation of $c_{i}$ is done by thinning the segmentation $S_{i-1}$ using the method presented by Lee 
in [8] and a sequential polynomial least-squares approximation of the $x-, y-$ and $z$-component of the skeleton voxels. In general a polynomial degree of four to six is sufficient.

The second phase in every iteration $i$ is the minimization of the energy function $U$ given the previous segmentation $S_{i-1}$ and the parametric centerline $c_{i}$. The objective function is derived from MRF theory [9]. The optimal solution is defined by the maximum of the probability function $P\left(S_{i}\right)=Z^{-1} \exp \left(-U\left(S_{i}\right)\right)$ with $Z$ being a normalization constant, $U\left(S_{i}\right)=\sum_{\boldsymbol{x}} V\left(s_{\boldsymbol{x}} \mid S_{i}\right)$ being the objective energy function and $s_{\boldsymbol{x}}$ being the state of the voxel $\boldsymbol{x}$. There are two different states: occupied $\left(s_{\boldsymbol{x}}=1\right)$ if the voxel is part of the spinal channel/cord and free $\left(s_{\boldsymbol{x}}=0\right)$ otherwise. Using $S_{i-1}$ as initialization we assume that we are within the area of attraction of the correct minimum 10. For this reason a local gradient descent strategy can be used for optimization. $S_{i}$ is set to $S_{i-1}$ initially. Then for all voxels $\boldsymbol{x}$ neighboring $S_{i}$ the energy for the occupied state $e_{1}$ and the energy for the free state $e_{0}$ is computed. If $e_{1}<e_{0}$ the voxel $\boldsymbol{x}$ is added to the segmented area in $S_{i}$. This is repeated until no more voxels change from state free to occupied. First this is done for the segmentation of the spinal channel. This segmentation is then used as initialization for the segmentation of the spinal cord.

The potential $V\left(s_{\boldsymbol{x}} \mid S_{i}\right)$ is composed by the following four parts. The first part of the potential is called smoothness prior as it controls the homogeneity of the segmentation result. It can be formulated as

$$
V_{s}\left(s_{\boldsymbol{x}} \mid S_{i}\right)=1.0-\frac{1}{\left|\mathcal{N}_{\boldsymbol{x}}\right|} \sum_{\left\{\boldsymbol{x}^{\prime} \in \mathcal{N}_{\boldsymbol{x}} \mid s_{\boldsymbol{x}^{\prime}}=s_{\boldsymbol{x}}\right\}} 1
$$

with $\mathcal{N}_{\boldsymbol{x}}$ being the neighborhood of $\boldsymbol{x}$ and $|\cdot|$ the cardinality. This means that $V_{s}\left(s_{\boldsymbol{x}} \mid S_{i}\right)$ is zero if all voxels in the neighborhood of $\boldsymbol{x}$ have the state $s_{\boldsymbol{x}}$. If all neighboring voxels have a different state than $s_{\boldsymbol{x}}$ the potential is one.

The second one uses knowledge about the intensity range of the spinal channel and cord. The intensities are modeled by normal distributions $N\left(\mu_{c}, \sigma_{c}^{2}\right)$ and $N\left(\mu_{o}, \sigma_{o}^{2}\right)$ with $\mu_{c}$ and $\sigma_{c}$ being the parameters of the spinal channel and $\mu_{o}, \sigma_{o}$ of the cord respectively. Thus, the resulting intensity potential $V_{v}$ of a voxel $\boldsymbol{x}$ can be formulated as

$$
V_{v}\left(s_{\boldsymbol{x}} \mid S_{i}\right)=(-1)^{s_{\boldsymbol{x}}+1}\left(\left|\mathcal{I}(\boldsymbol{x})-\mu_{\{c, o\}}\right|-2 \sigma_{\{c, o\}}\right) /\left(2 \sigma_{\{c, o\}}\right)
$$

where $\mathcal{I}$ is the MRI volume of the spine. For $s_{\boldsymbol{x}}=1$ the potential has the minimum - 1 if $\mathcal{I}(\boldsymbol{x})=\mu_{\{c, o\}}$, it is zero if $\mathcal{I}(\boldsymbol{x})=\mu_{\{c, o\}} \pm 2 \sigma_{\{c, o\}}$ and positive if the signal intensity differs more than $2 \sigma_{\{c, o\}}$ from $\mu_{\{c, o\}}$. If the voxel's state is $s_{\boldsymbol{x}}=0$, the maximum is 1 and falls down linearly to $-\infty$. Consequently, voxels having an intensity between $\mu_{\{c, o\}} \pm \sigma_{\{c, o\}}$ are preferred to be within the segmentation; voxels with intensities that differ more than $\sigma_{\{c, o\}}$ from the mean $\mu_{\{c, o\}}$ tend to belong to the background.

The third part of the potential utilizes the relative position of the voxel to the current centerline estimation $c_{i}$. With $d_{c}(\boldsymbol{x})$ being the Euclidean distance from the voxel $\boldsymbol{x}$ to the centerline $c_{i}$ the potential $V_{c}$ can be written as 

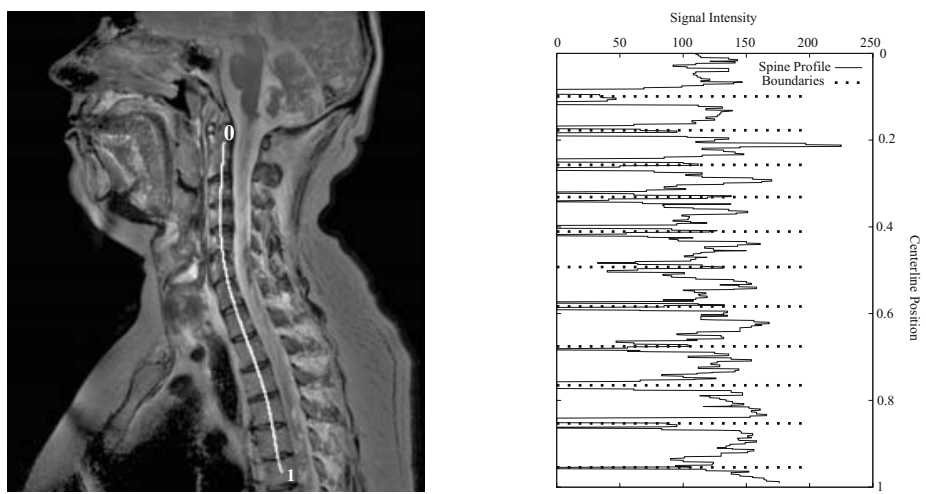

Fig. 2. The left image shows a $12 \mathrm{~mm}$ thick MIP in the spinal region including the computed centerline through the vertebra (white line). The data set shown is preprocessed. This centerline is used to compute the boundary positions of the vertebra. In the righthand plot the signal intensities along the centerline are shown. Additionally the estimated boundaries of the vertebra are illustrated (dotted lines). The computed threshold was $\theta=70.3$.

$$
V_{c}\left(s_{\boldsymbol{x}} \mid S_{i}\right)=(-1)^{s_{\boldsymbol{x}}+1}\left(d_{c}(\boldsymbol{x})-r\right) / r
$$

where $r / 2$ is the average radius of the spinal channel/cord. As a result $V_{c}\left(s_{\boldsymbol{x}}=\right.$ $\left.1 \mid S_{i}\right)<V_{c}\left(s_{\boldsymbol{x}}=0 \mid S_{i}\right)$ for voxels that are closer than $r / 2$ to the approximated center line $c_{i}$ and $V_{c}\left(s_{\boldsymbol{x}}=1 \mid S_{i}\right)>V_{c}\left(s_{\boldsymbol{x}}=0 \mid S_{i}\right)$ if the distance is larger. The minimal value of $V_{c}$ for the occupied state is -1 ; the maximal value of the potential for $s_{\boldsymbol{x}}=0$ is 1 .

Finally, the last part of the potential uses the scalar product between the propagation direction of the segmentation and the gradient of the image intensities. As propagation direction we use the gradient of the segmentation image $S_{i}$ at the voxel $\boldsymbol{x}$. Thus, the potential can be defined as

$$
V_{g}\left(s_{\boldsymbol{x}} \mid S_{i}\right)=(-1)^{s_{\boldsymbol{x}}}\left(1-\left|(\nabla \mathcal{I}(\boldsymbol{x}))^{T} \nabla S_{i}(\boldsymbol{x})\right| / m\right)
$$

where $m$ is the maximal tolerable magnitude of the gradient. If the gradients are aligned parallel or anti-parallel or if the image gradient is zero, the potential for $s_{\boldsymbol{x}}=1$ has its minimal value -1 and its maximal value 1 for $s_{\boldsymbol{x}}=0$.

\subsection{Labeling of the Vertebra and Visualization}

In order to label the vertebra within the images, we compute an intensity profile $p$ on the ventral side of the estimated centerline. Then we apply a threshold $\theta$ to the computed profile $p$. From this an initial guess about the positions of the vertebra is computed. Finally, this guess is refined using the average distances between the vertebra. The profile as well as the estimated boundaries of the vertebra are illustrated in Figure 2, As there is no slice where the whole centerline through the vertebra can be seen we use a $12 \mathrm{~mm}$ thick Maximum Intensity Projection 
(MIP) to be able to cover the whole spine in the illustration. The original slice thickness was $1 \mathrm{~mm}$.

For the visualization, the computed centerline is approximated by splines. Using the parametric approximation we can compute MPRs that are orthogonal to the backbone for every position of the spinal channel/cord. An illustration of the presentation of the MPRs is shown in Figure 3.

\section{Results}

Data Sets. All data sets were acquired during clinical routine. In total we used 20 3-D MRI SPACE data sets from the spine including ten volumes showing the upper spine and ten data sets covering the lower spine. All images were acquired with a repetition time of $\mathrm{TR}=1000 \mathrm{~ms}$ and an echo time of $\mathrm{TE}=130 \mathrm{~ms}$. The volumes had a isotropic in-plane resolution between $0.8 \mathrm{~mm} \times 0.8 \mathrm{~mm}$ and $1.3 \mathrm{~mm} \times 1.3 \mathrm{~mm}$ and a slice thickness of $1 \mathrm{~mm}$. The image matrix had a size of $384 \times 384$. Every scan consists of 60 up to 160 slices. All used data sets are publicly available at our homepage1.

Evaluation Method. The whole processing chain was implemented in $\mathrm{C}++$ and integrated into the ITK Framework (http://www.itk.org). For a better presentation of the results and to increase the usability for radiologists everything was integrated into the medical visualization platform InSpace3D. The experiments were performed on a $2.00 \mathrm{GHz}$ Intel Core2 CPU with $2 \mathrm{~GB}$ RAM. The whole processing chain took about 5-20s depending on the size and the bending of the backbone.

The focus of this work is an easy-to-use framework for CAA of anomalies in the scoliotic spine. For this reason it is important that radiologists have an orthogonal view onto every vertebra. Thus, we use the following quality measure for evaluation. First, for every vertebra $v$ within the images, a medical expert defines a ground truth plane with normal $\boldsymbol{n}_{\boldsymbol{g}}{ }^{v}$. Then, the corresponding planes with normal $\boldsymbol{n}_{\boldsymbol{a}}{ }^{v}$ are computed using the proposed segmentation method. In order to measure the distance between the corresponding planes we use the angle

$$
d_{v}=\arccos \left|\left(\boldsymbol{n}_{\boldsymbol{g}}{ }^{v}\right)^{T} \cdot \boldsymbol{n}_{\boldsymbol{a}}{ }^{v}\right|
$$

between the normal vectors. The range of $d_{v}$ is $\left[0^{\circ}, \ldots, 90^{\circ}\right]$. If both planes are aligned perfectly parallel or anti-parallel the angle between the normal vectors is $d_{v}=0$ degree. If, on the other hand, the corresponding planes are orthogonal, $d_{v}=90$. The quality $q$ of the proposed segmentation method is computed by

$$
q=\frac{1}{V} \sum_{i=1}^{V} d_{i}
$$

with $V$ being the number of ground truth planes. It reflects the mean angular deviation of the ground truth to the automatically computed planes.

\footnotetext{
${ }^{1}$ http://www5.informatik.uni-erlangen.de/ ${ }^{\sim}$ spine/
} 


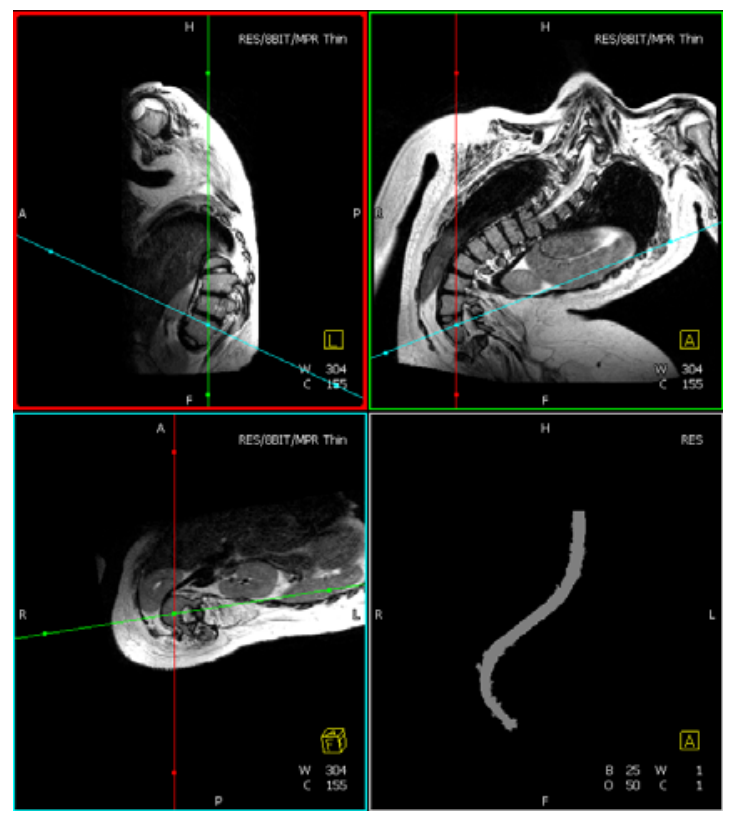

Fig. 3. The figure shows the presentation of the computed MPRs. The upper two images show the sagittal plane and the coronal plane respectively. The second row shows the plane orthogonal to the spinal cord. Finally, the result of the segmentation of the spinal cord is presented in a $3-\mathrm{D}$ view (lower right).

Evaluation Results. In total planes through $V=181$ different vertebra were defined by the radiologist. Using the proposed quality measure for our segmentation method yielded a mean distance of $q=5.74^{\circ}$ with a standard deviation of $\sigma_{q}=6.13^{\circ}$. The minimal deviation was $0.42^{\circ}$ and the maximal deviation was 20.15 degrees (lower spine: $\mu_{l}=6.98^{\circ}, \sigma_{l}=7.69, \min _{l}=0.83^{\circ}, \max _{l}=20.15^{\circ}$, upper spine: $\left.\mu_{u}=5.14^{\circ}, \sigma_{u}=5.22, \min _{u}=0.42^{\circ}, \max _{u}=17.19^{\circ}\right)$. If it is assumed that an average vertebra has a size of about $30 \times 30 \times 20 \mathrm{~mm}^{3}$ this means that there is a distance between the two planes of less than $2 \mathrm{~mm}$ at the border of an average vertebra.

Additionally, a second radiologist defined $V=61$ planes through vertebra that were also labeled by the first radiologist. These planes were used to compute the inter-observer variability of both radiologists. This resulted in a mean angular deviation of $q_{o}=2.94^{\circ}\left(\sigma_{q_{o}}=1.99^{\circ}, \min =0.48^{\circ}, \max =9.38^{\circ}\right)$.

The results show that the proposed algorithm works very reliable for the upper spine. Especially in the lower lumbar area and the pelvic region the results get slightly worse. The reason for this is that the medulla ends in this region and separate nerve cords are left. Thus, there is a higher probability that the segmentation follows these cords away from the backbone. In clinical routine this is not a big problem, as these regions are irrelevant for diagnostics in general. 


\section{Discussion and Conclusion}

We presented a novel approach for the segmentation of the spinal cord based on MRF theory. The segmentation is used to compute planes orthogonal to the vertebra column for CAA of anomalies in the scoliotic spine. The advantage of our method is that we do not use any segmentation of the vertebra itself or information about their relative positioning. Thus, even an extreme bending of the spine or pathologic changes of the vertebra structure can be compensated easily. Further on, the method presented works on 3-D volumes and is not restricted to a good visible coverage of the spine in a single slice. Additionally, no training step is required. Thus, the method can easily adapt if the acquisition protocol changes or other modalities like CT are used.

The proposed framework enables the radiologist to easily assess anomalies in the scoliotic spine. The errors in orientation observed are small enough for clinical usage. Furthermore, the majority of false centerline estimations occurs in the pelvic region of the spine that is only of little diagnostic interest. The observed errors can further be reduced by post-processing like a rough segmentation of the vertebra using the approximated centerline to improve their pose estimation.

\section{References}

1. Lewonowski, K., King, J.D., Nelson, M.D.: Routine use of magnetic resonance imaging in idiopathic scoliosis patients less than eleven years of age. Spine 17(Suppl. 6), 109-116 (1992)

2. Gupta, P., Lenke, L.G., Bridwell, K.H.: Incidence of neural axis abnormalities in infantile and juvenile patients with spinal deformity. is a magnetic resonance screening necessary? Spine 23, 206-210 (1998)

3. Peng, Z., Zhong, J., Wee, W., Lee, J.: Automated vertebra detection and segmentation from the whole spine MR images. In: IEEE-EMBS, New York City, USA, pp. 2527-2530. IEEE, Los Alamitos (2006)

4. McIntosh, C., Hamarneh, G.: Spinal crawlers: Deformable organisms for spinal cord segmentation and analysis. In: Larsen, R., Nielsen, M., Sporring, J. (eds.) MICCAI 2006. LNCS, vol. 4190, pp. 808-815. Springer, Heidelberg (2006)

5. Coulon, O., Hickman, S.J., Barker, G.J., Miller, D.H., Arridge, S.R.: Quantification of spinal cord atrophy from magnetic resonance images via a b-spline active surface model. Magn. Reson. Med. 47(6), 1176-1185 (2002)

6. Brinkmann, B.H., Manduca, A., Robb, R.A.: Optimized homomorphic unsharp masking for MR grayscale inhomogeneity correction. IEEE Trans. Med. Imag. 17(2), 161-171 (1998)

7. Jäger, F., Hornegger, J.: Nonrigid registration of joint histograms for intensity standardization in magnetic resonance imaging. IEEE Trans. Med. Imag. 28(1), 137-150 (2009)

8. Lee, T.C., Kashyap, R.L., Chu, C.N.: Building skeleton models via 3-d medial surface/axis thinning algorithms. CVGIP 56(6), 462-478 (1994)

9. Li, S.Z.: Markov Random Field Modeling in Image Analysis. Computer Science Workbench. Springer, Tokyo (2001)

10. Fetita, C.L., Prêteux, F.J.: Quantitative 3d ct bronchography. In: ISBI, Washington, DC, USA, pp. 221-224. IEEE, Los Alamitos (2002) 